Research Paper

\title{
Survival and Symptom Relief after Palliative Radiotherapy for Esophageal Cancer
}

\author{
Julia Welsch1,Philipp Günther Kup ${ }^{1}$, Carsten Nieder2,3, Veria Khosrawipour ${ }^{4}$, Helmut Bühler1, Irenäus A. \\ Adamietz ${ }^{1,5,6}$, Khashayar Fakhrian ${ }^{1,5} \bowtie$ \\ 1. Department of Radiation Oncology, Marien Hospital Herne, Ruhr-University Bochum, Herne, Germany. \\ 2. Department of Oncology and Palliative Medicine, Nordland Hospital Bodø, Norway \\ 3. Department of Clinical Medicine, Faculty of Health Sciences, University of Tromsø, Norway. \\ 4. Department of Surgery, Marien Hospital Herne, Ruhr-University Bochum, Herne, Germany. \\ 5. Department of Radiation Oncology, Sankt Josef Hospital Bochum, Ruhr-University Bochum, Bochum, Germany. \\ 6. Department of Radiation Oncology, Ev-Krankenhaus Witten, Academic Hospital of the Witten/Herdecke University, Witten, Germany. \\ $\square$ Corresponding author: Dr. Khashayar Fakhrian, Vice Chair, Department of Radiation Oncology, Ruhr-University Bochum, Marien Hospital Herne, \\ Hölkeskampring 40, 44625 Herne, Germany. Telephone: (+)49 (0)2323 499-1531, Fax: (+)49 (0)2323 499-306, Email: khfmed@yahoo.com
}

( ) Ivyspring International Publisher. Reproduction is permitted for personal, noncommercial use, provided that the article is in whole, unmodified, and properly cited. See http://ivyspring.com/terms for terms and conditions.

Received: 2015.08.25; Accepted: 2015.11.01; Published: 2016.01.01

Abstract

Purpose: The aim of this study was to assess the 6-months dysphagia-free survival, improvement in swallowing function, complication rate, and overall survival in patients with incurable esophageal cancer treated with palliative radiotherapy.

Methods: We retrospectively reviewed data from 139 patients (median age 72 years) with advanced/recurrent incurable esophageal cancer, who were referred to 3 German radiation oncology centers for palliative radiotherapy between 1994 and 2014. Radiotherapy consisted of external beam radiotherapy (EBRT) with $30-40.5 \mathrm{~Gy} / 2.5$ - 3 Gy per fraction, brachytherapy alone (BT) with 15 - 25 Gy/5 - 7Gy per fraction/weekly and EBRT + BT (30 - 40.5 Gy plus 10 - 14 Gy with BT) in 65, 46, and 28 patients, respectively. Dysphagia-free survival (Dy-PFS) was defined as the time to worsening of dysphagia for at least one point, a new loco-regional failure or death of any cause.

Results: Median follow-up time was 6 months (range 1-6 months). Subjective symptom relief was achieved in $72 \%$ of patients with median response duration of 5 months. The 1 -year survival rate was $30 \%$. The 6-months Dy-PFS time for the whole group was $73 \pm 4 \%$. The 6-months Dy-PFS was $90 \pm 4 \%$ after EBRT, $92 \pm 5 \%$ after EBRT + BT and $37 \pm 7 \%$ after BT, respectively $(p<0.001)$. Five patients lived for more than 2 years, all of them were treated with EBRT \pm BT. Ulceration, fistula and stricture developed in 3, 6 and 7 patients, respectively.

Conclusions: Radiotherapy leads to symptom improvement in the majority of patients with advanced incurable esophageal cancer. The present results favor EBRT \pm BT over BT alone. Due to the retrospective nature of this study, imbalances in baseline characteristics might have contributed to this finding, and further trials appear necessary.

Key words: Esophageal cancer, Palliation, External beam radiation therapy, Brachytherapy, Dysphagia-free survival, Complications

\section{Introduction}

More than $50 \%$ of esophageal cancer patients present with locally advanced stage or distant metastases with tumor related symptoms and/or poor general condition $[1,2]$. The most common cancer-related complication in these patients is dysphagia with an increasing rate as the disease progresses, leading to nutritional compromise, pain, reduced performance status and deterioration of quality of life $[3,4]$. Many of these patients with severe clinical symptoms are not fit enough to undergo surgical re- 
section or curative radiochemotherapy $[5,6]$. The latter approaches are not suitable for patients with distant metastases either [7]. In symptomatic patients, long-term palliation of dysphagia is an important goal of therapy. Various palliative treatment modalities have been used in an attempt to relieve dysphagia and improve quality of life for the remaining life time. These include stent placement, dilation, external beam radiation therapy (EBRT), brachytherapy, chemotherapy, laser treatment, photodynamic therapy or ablation $[8,9]$. Of those approaches endoluminal stenting, radiation therapy, or a combination of both are most commonly used [10]. Esophagectomy for palliation is not recommended due to the associated high morbidity and mortality rate. Thermal and chemical ablative therapies provide acceptable dysphagia palliation but have an increased requirement for re-interventions and for adverse effects. Chemotherapy alone for symptom relief might be possible in some patients, but it takes several weeks to achieve this effect. Patients in poor general condition are not fit enough to undergo effective chemotherapy [11]. Development of distant metastases during the course of disease is common. Thus, oncologists often chose local treatment options for relief of initial local symptoms and postpone chemotherapy for the treatment of systemic disease. Radiotherapy is a potent local treatment option, which can provide symptom relief and prolongation of survival. Its effects were the subject of the present retrospective study. The primary endpoint of the study was the 6-month dysphagia progression-free survival (6-mo Dy-PFS). Secondary endpoints were overall survival, time to relapse of dysphagia to the pretreatment status (or worse), time to worsening of dysphagia by at least one point, and complication rate.

\section{Methods and Material}

\section{Patients}

We retrospectively reviewed data from 189 patients with advanced/recurrent esophageal cancer, who were referred to one of three German radiation oncology centers (Bochum, Herne, Witten) for palliative radiotherapy from 1994 to 2014. Patients with histologically confirmed squamous cell or adenocarcinoma of the esophagus, considered too advanced or medically unsuitable for radical treatment (with or without metastatic disease), symptomatic with dysphagia score of 1-4 $(0=$ no dysphagia, Table 1$)$ were eligible. Patients were excluded for the following reasons: previous radiochemotherapy, presence of an esophageal fistula, simultaneous other primary cancer. One-hundred thirty nine patients fulfilled the inclusion criteria and were enrolled in this study. Pa- tients' characteristics are summarized in Table 2. The pre-treatment evaluation included a medical history and physical examination, complete blood count, computed tomography of the neck/chest/abdomen and an esophagogastroscopy and endosonography, if technically possible. A bronchoscopy was carried out if there was a suggestion on computed tomography or clinical evidence of airway involvement.

Table 1. Dysphagia scoring scale

\begin{tabular}{ll}
\hline 0 & Able to consume a normal diet \\
\hline 1 & Dysphagia with certain solid foods \\
2 & Able to swallow semi-solid soft foods \\
3 & Able to swallow liquids only \\
4 & Unable to swallow saliva (complete dysphagia) \\
\hline
\end{tabular}

Table 2. Characteristics of all patients

\begin{tabular}{ll}
\hline Characteristics No. (\%) & All patients \\
& N $=139$ \\
\hline Gender & $31(22)$ \\
Female & $108(78)$ \\
Male & $70(48-85)$ \\
Age, mean (range), y & \\
Histology & $101(73)$ \\
Squamous cell carcinoma & $38(27)$ \\
Adenocarcinoma & \\
Localisation & $60(43)$ \\
Suprabifurcal & $48(35)$ \\
Infrabifurcal & $31(22)$ \\
Overlapping & \\
Length & $122(88)$ \\
$\leq 8$ cm & $17(12)$ \\
$>8$ cm & \\
Lymph node metastases, cN+ & $116(84)$ \\
Yes & $23(17)$ \\
No & \\
ECOG-PS at palliative treatment & $39(28)$ \\
$0-1$ & $100(72)$ \\
$2-3$ & \\
Treatment & $65(47)$ \\
EBRT & $46(33)$ \\
BT & $28(20)$ \\
EBRT+BT & \\
Dysphagia score & $0(0)$ \\
0 & $42(30)$ \\
1 & $60(43)$ \\
2 & $30(22)$ \\
3 & $7(5)$ \\
4 &
\end{tabular}

ECOG-PS: Eastern Cooperative Oncology Group performance status; EBRT: external beam radiotherapy; BT: brachytherapy

\section{Treatment}

Radiotherapy consisted of EBRT with 30-40.5 Gy/2.5-3 Gy per fraction, 5 days a week in 65 patients. The target volume consisted of the primary esophageal tumor with margins of $1-3 \mathrm{~cm}$ in the cranial caudal direction and $1 \mathrm{~cm}$ circumferentially. Endoluminal treatment was administered by a high dose rate (HDR) afterloading device with an iridium ${ }^{192}$ 
source. For the treatment a blind ending gastric tube of $10 \mathrm{~mm}$ was passed through the tumor stenosis. The applicator with a centimeter scaled dummy chain for determination of the irradiated length was then inserted. The irradiation length included the tumor stenosis with a safety margin of $1-2 \mathrm{~cm}$ above and below. The single dose of each HDR treatment was 5-7 Gy specified to $1 \mathrm{~cm}$ from the applicator surface. Brachytherapy (BT) alone was performed in 46 patients with a total dose of 15-25 Gy/5 Gy per fraction/weekly (Table 3). Twenty-eight patients underwent EBRT (30 - $40 \mathrm{~Gy}$ ) plus 1 to 3 fractions of BT with a dose of 5 - 7 Gy per fraction/weekly. A stent was placed in 18 patients prior to and in 25 patients after radiotherapy. Chemotherapy was administered to 18 patients prior to and 39 patients after radiotherapy.

Table 3. Baseline characteristics for different treatment groups

\begin{tabular}{llllll}
\hline $\begin{array}{l}\text { Characteristics N } \\
(\%)\end{array}$ & $\begin{array}{l}\text { All Patients } \\
\mathrm{n}=139\end{array}$ & $\mathrm{BT}$ & EBRT & BT+EBRT & $\mathrm{p}$ \\
\hline $\begin{array}{l}\text { Gender } \\
\text { Female }\end{array}$ & $31(22)$ & $8(17)$ & $18(28)$ & $5(18)$ & 0.426 \\
$\begin{array}{l}\text { Male } \\
\text { Age, Median } \\
\text { (range) }\end{array}$ & $108(78)$ & $38(83)$ & $47(72)$ & $23(82)$ & \\
ECOG-PS & $72(48-85)$ & $73(61-85)$ & $69(57-81)$ & $69(48-81)$ & $<0.001$ \\
0-I & & & & & \\
$\begin{array}{l}\text { II-III } \\
\text { Tumor length }\end{array}$ & $39(28)$ & $8(17)$ & $21(32)$ & $10(36)$ & 0.130 \\
$\begin{array}{l}\text { 1-8cm } \\
>8 \mathrm{~cm}\end{array}$ & $100(72)$ & $38(82)$ & $44(68)$ & $18(64)$ & \\
\hline
\end{tabular}

ECOG-PS: Eastern Cooperative Oncology Group performance status; EBRT: external beam radiotherapy; BT: brachytherapy

\section{Dysphagia scoring, follow-up and toxicity analysis}

Dysphagia was measured on a five-point dysphagia scale (Table 1). Acute treatment-related toxicity was graded according to the National Cancer Institute's Common Terminology Criteria for Adverse Events, version 3.0 (CTC 3.0), except for dysphagia. For the dysphagia, the above mentioned dysphagia score was used. Patients were assessed weekly during the treatment and each 2 - 4 weeks thereafter. At week 8-10, all patients underwent a reassessment of the local disease with either an endoscopy and/or CT. This was repeated at the time of dysphagia progression to confirm whether progression was secondary to disease or stricture. After finishing radiotherapy patients were followed for six months until symptomatic or luminal disease progression, and every 2 months thereafter if clinically feasible. At each visit, patients were assessed with a medical history, physical examination, dysphagia score, toxicity score, weight and performance status (PS).

\section{Statistical methods}

Statistical analyses were performed using the
SPSS software (version 20.0). Overall survival (OS) was defined as death from any cause, dysphagia-free survival (Dy-PFS) was defined as the time to worsening of dysphagia by at least one point, loco-regional failure or death of any cause.

All time estimates began with the 1st day after start of radiation therapy to the date of first failure or the date of last clinical visit, whichever occurred first.

OS and PFS probabilities were estimated using Kaplan-Meier analysis. Unadjusted associations between continuous variables and toxicities $\geq$ grade 2 were assessed by Wilcoxon Rank-Sum test or Kruskal-Wallis test. Unadjusted associations between categorical variables and toxicities were assessed by Fisher's exact test.

\section{Results}

Median follow-up time was 6 months (range 1-6 months). A dysphagia score of $1,2,3$ or 4 was observed in $30 \%, 43 \%, 22 \%$ and $5 \%$ of patients at the start of the treatment, respectively. Longitudinal changes are displayed in Figures 1-3. The 6-months Dy-PFS probability for the whole group was $73 \pm 4 \%$ (Fig. 4). The 6-months Dy-PFS was $90 \pm 4 \%$ after EBRT, $92 \pm 5 \%$ after EBRT + BT and $37 \pm 7 \%$ after BT, respectively $(p<0.001$, Fig. 5). Subjective symptom relief was achieved in $72 \%$ of all patients with median response duration of 5 months. The median time period to dysphagia improvement for at least one point was 26 weeks for patients who were treated with EBRT \pm BT and 16 weeks for patients who were treated with BT alone (Fig. 2-3). A worsening back to the initial dysphagia score or worse was observed in $35 \%$ after BT, $8 \%$ after EBRT and 7\% after EBRT + BT, respectively. The 1- and 2-year OS rates were $30 \pm 4 \%$ and $4 \pm 2 \%$, respectively with a median overall survival time of 10 months (Fig. 6). Five patients lived more than 2 years, all of them were treated with $\mathrm{EBRT} \pm \mathrm{BT}$.

\section{Treatment sequelae}

There were no grade 5 toxicities. Mucositis $\geq$ grade $2(39 \%)$, temporary worsening of general condition $(29 \%)$ and temporary increase of pain $(25 \%)$ were the most common acute toxicities of the treatment. Bacterial pneumonia and hematologic toxicity $\geq$ grade 2 were observed in $6 \%$ and $4 \%$ of the patients, respectively. There were 3 ulcerations in the context of persistent or recurrent tumor, 2 patients were treated with stent + EBRT and 1 with induction chemotherapy + BT. In 6 patients a fistula formation was observed, 2 were treated with BT, 3 with stent + EBRT and 1 patient with induction chemotherapy + stent + EBRT. In 7 patients esophageal strictures were observed, requiring repeated bouginage. 


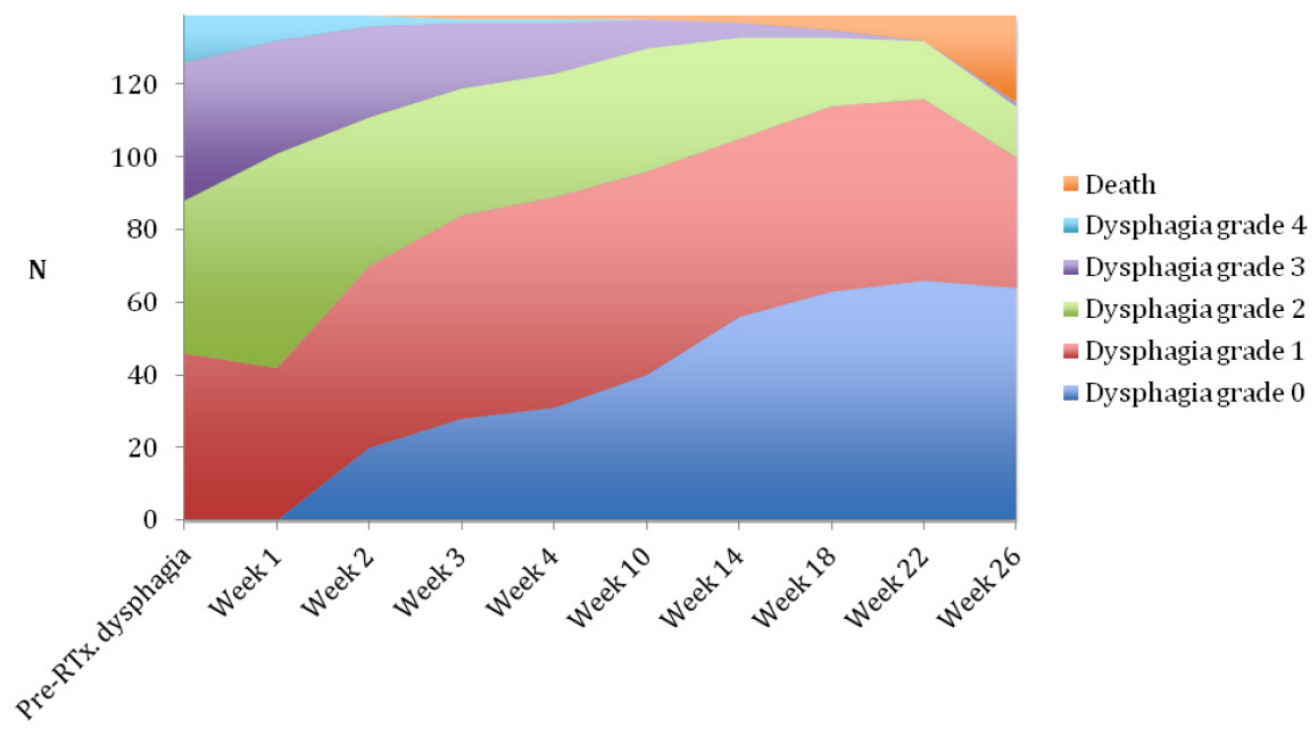

Fig 1. Changes in dysphagia score over time in all patients.

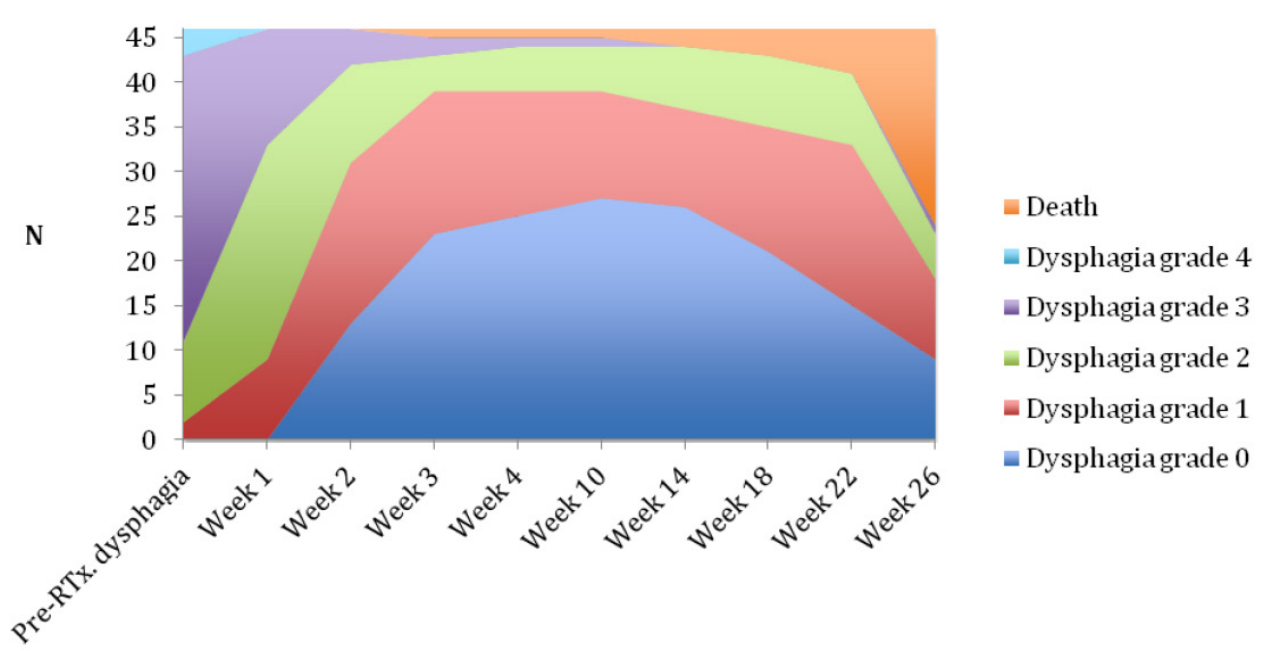

Fig 2. Changes in dysphagia score over time in the brachytherapy group.

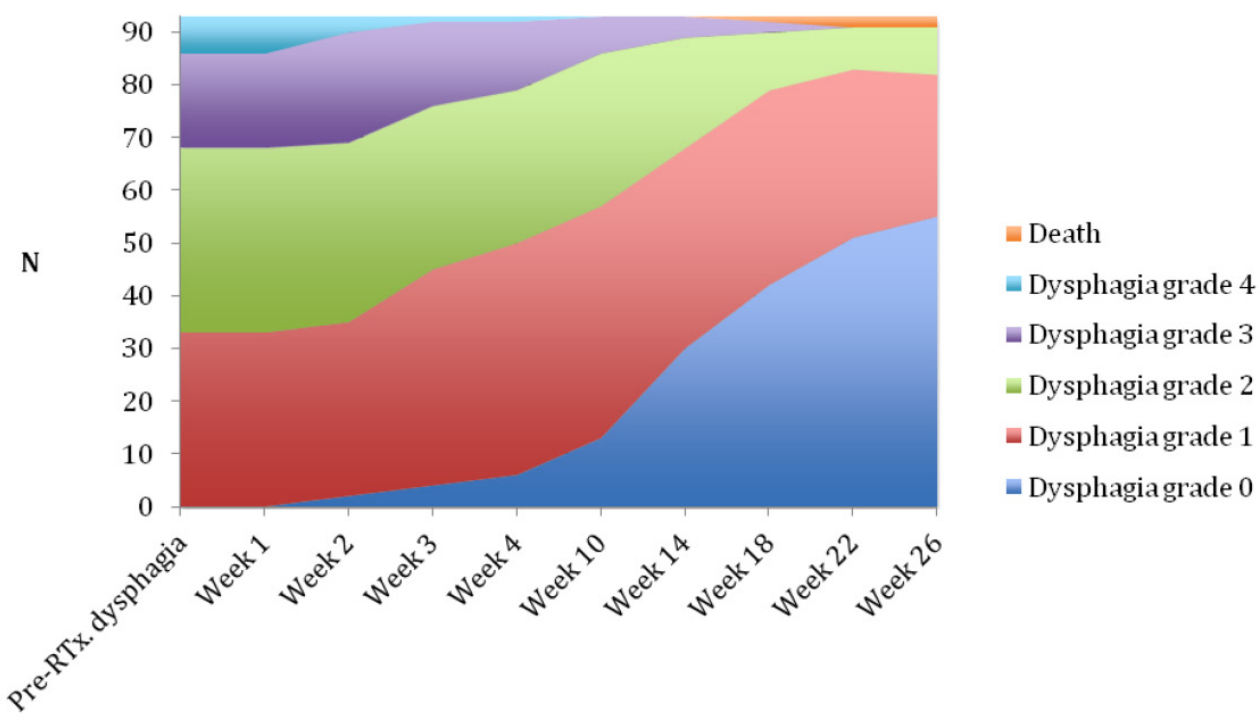

Fig 3. Changes in dysphagia score over time in the EBRT \pm brachytherapy group. 


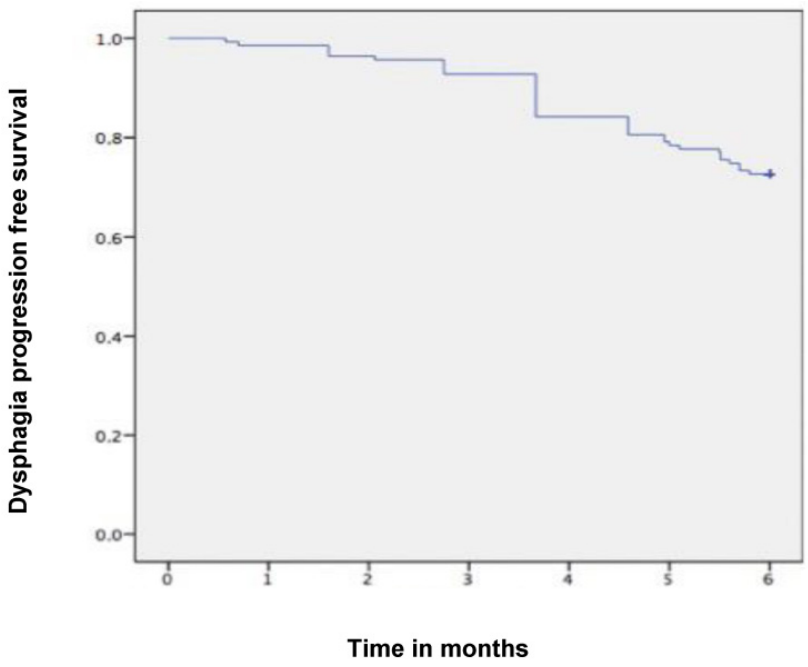

Fig. 4 Kaplan-Meier curves for dysphagia progression free survival in the whole cohort.

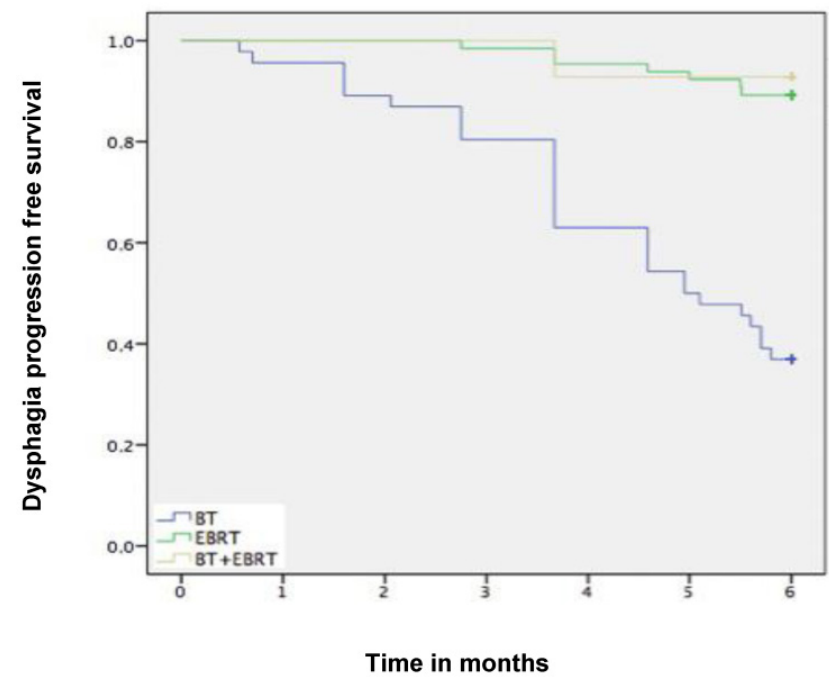

Fig. 5 Kaplan-Meier curves for dysphagia progression free survival in the subgroups.

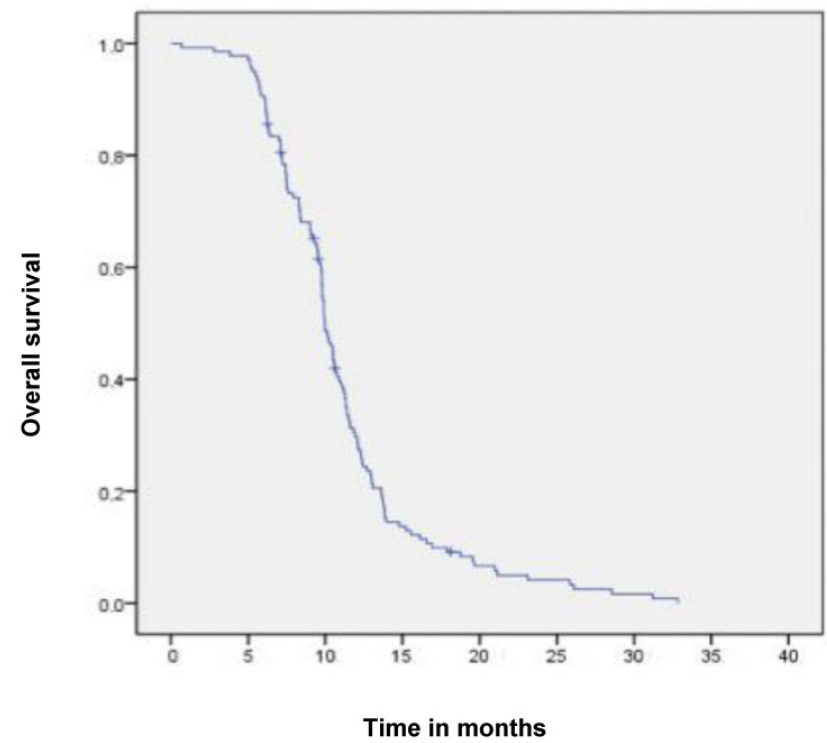

Fig. 6 Kaplan-Meier curves for overall survival in the whole cohort

\section{Discussion}

We report our multicenter 20-year experience with 139 incurable esophageal cancer patients treated for symptom relief with palliative RT. The overall treatment concept was individualized and included various combinations of stent placement, chemotherapy, and RT. Performance status, local tumor extension/obstruction, distant metastases and comorbidity were assessed before the overall concept was chosen. The optimal treatment regimen for palliation of dysphagia in locally advanced esophageal cancer is still controversially discussed [12]. However, effective symptom relief has been reported in many randomized and non-randomized clinical trials. It has been shown that stent placement alone was inferior to stent plus BT and stent loaded with radioactive seeds [13, 14]. A further randomized trial ( $\mathrm{n}=84$ patients) compared stent alone to stent and EBRT, 30 Gy in 10 fractions [15]. Dysphagia scores improved significantly in both groups following stent insertion. However, dysphagia relief was more sustained after additional EBRT (7 vs. 3 months, $p=0.002$ ). Overall median survival was significantly longer after additional EBRT (180 vs. 120 days, $p=0.009)$. There was no treatment-related mortality, and the incidence of complications was similar in the two groups. A fourth randomized study ( $\mathrm{n}=219$ patients) examined whether the combination of HDR BT and EBRT is superior to HDR BT alone for the palliation of esophageal cancer [16]. All patients received two fractions of HDR BT within 1 week (8 Gy prescribed at $1 \mathrm{~cm}$ from source center). Patients randomized to EBRT received 30 Gy in 10 fractions. The primary outcome was dysphagia-relief experience (DRE). Median survival was 6 months (18\% survival rate at 1 year). DRE was significantly improved with combined therapy, for an absolute benefit of $+18 \%$ at 200 days from randomization $(p=0.019)$. In longitudinal regression analyses, scores for dysphagia, odynophagia, regurgitation, chest pain and performance status were all significantly improved. In contrast, weight, toxicities and overall survival were not different between study arms. Together, these data from 4 individual studies suggest that combined therapy with stent + BT, stent + EBRT or BT + EBRT is well tolerated and relatively safe. Yet not all possible combinations have been compared in randomized trials.

Patients with distant metastatic stage IVB esophageal cancer with dysphagia due to the primary lesion might best be treated with chemotherapy plus local measures [17]. The authors investigated 40 patients. Treatment consisted of two courses of chemotherapy (5-fluorouracil and cisplatin) and concurrent irradiation of $40 \mathrm{~Gy}$ in 20 fractions to the esophageal primary tumor. Dysphagia score improved in $75 \%$ of 
the patients. Seventeen of the 20 patients (85\%) who had required nutritional support at baseline improved their oral intake to no longer need the support, in a median time of 43 days. Disease control rate of the primary lesion was $95 \%$, including 12 patients (30\%) who achieved a complete response. The median survival was 10 months, and the 1-year-survival rate was $45 \%$. Major toxicities (grade 3 or 4 ) involved hemoglobin $(23 \%)$, leukocytes (15\%), neutrophils (20\%), anorexia (10\%), nausea (3\%), esophageal perforation $(5 \%)$ and febrile neutropenia (3\%). Two patients $(5 \%)$ died within 30 days of terminating radiotherapy.

Murray et al. performed a retrospective study, which shares important similarities with ours [18]. They analyzed data from 148 patients with histologically proven esophageal cancer, who were unsuitable for radical treatment (2005-2010). The median age of their patients was 74 years (range, 31-91). Forty-nine percent of patients were WHO PS 2 or 3, 70\% had adenocarcinoma (only $27 \%$ in our study), $58 \%$ had locally advanced disease, and $28 \%$ had metastatic disease. Ninety-three percent of patients complained of dysphagia prior to radiotherapy, and $6 \%$ had a stent placement. Eighty-nine percent of patients received a 2-D planned dose of 20 Gy in 5 fractions, i.e. a significantly lower dose than in most other studies discussed here, also in terms of biologically effective dose. Following radiotherapy, $75 \%$ of patients experienced an improvement in dysphagia and 25\% of patients gained weight. Dy-PFS was not analyzed. Twenty-six percent of patients required subsequent insertion of an esophageal stent and a further 3\% received retreatment with radiotherapy. The median stent (and retreatment)-free survival was 4.9 months. In our study $18 \%$ of patients had subsequent stent insertion and median response duration after RT was 5 months. Murray et al. reported median overall survival of 6.1 months (1-year survival 29\%), compared to 10 months (30\%). These data suggest that short-course radiotherapy could be considered in patients with limited survival expectation, e.g. those with poor PS and/or widespread metastatic disease. Important caveats exist regarding inter-study comparisons, because inhomogeneous populations were included, comprised of patients with or without distant metastases, good or poor PS etc. Limitations regarding our own study also include patient selection for the different treatment modalities and group sizes. In line with the randomized IAEA study in squamous cell cancer [16], our data suggest that BT alone might not be the preferred RT option. Excellent palliation rates were observed after EBRT with or without BT, i.e. 6-months Dy-PFS of $90 \pm 4 \%$ after EBRT and $92 \pm 5 \%$ after EBRT + BT. A worsening back to the initial dysphagia score or worse was uncommon $(8 \%$ after EBRT and 7\% after EBRT + BT, compared to 35\% after BT alone).

\section{Conclusion}

Radiotherapy leads to symptom improvement in about $70 \%$ of patients with esophageal cancer. Considering longer symptoms relief period and progression-free survival EBRT \pm BT appears superior to BT alone. However, the present results were not obtained in the context of a randomized trial.

\section{Competing Interests}

The authors have declared that no competing interest exists.

\section{References}

1. Torre LA, Bray F, Siegel RL, Ferlay J, Lortet-Tieulent J, Jemal A. Global cancer statistics, 2012. CA Cancer J Clin. 2015;65:87-108.

2. Parkin DM, Bray F, Ferlay J, Pisani P. Estimating the world cancer burden: Globocan 2000. Int J Cancer. 2001;94:153-156.

3. Cohen DJ, Leichman L. Controversies in the treatment of local and locally advanced gastric and esophageal cancers. J Clin Oncol. 2015;33:1754-1759.

4. Shah MA. Update on metastatic gastric and esophageal cancers. J Clin Oncol. 2015;33:1760-1769.

5. Fakhrian K, Ordu AD, Lordick F, et al. Long-term outcomes of trimodality treatment for squamous cell carcinoma of the esophagus with cisplatin and/or 5-FU: more than 20 years' experience at a single institution. Strahlenther Onkol. 2014;190:1133-1140.

6. Ordu AD, Nieder C, Geinitz H, et al. Radio(chemo)therapy for locally advanced squamous cell carcinoma of the esophagus: long-term outcome. Strahlenther Onkol. 2015;191:153-160.

7. Fakhrian $\mathrm{K}$, Ordu AD, Nieder C, et al. Outcomes of patients with squamous cell carcinoma of esophagus who did not receive surgical resection after neoadjuvant radiochemotherapy. Tumori. 2015;101:263-267.

8. Dai Y, Li C, Xie Y, et al. Interventions for dysphagia in oesophageal cancer. Cochrane Database Syst Rev. 2014 Oct 30;10:CD005048.

9. Weigel TL, Frumiento C, Gaumintz E. Endoluminal palliation for dysphagia secondary to esophageal carcinoma. Surg Clin North Am. 2002;82:747-761.

10. Siersema PD, Dees J, van Blankenstein M. Palliation of malignant dysphagia from oesophageal cancer. Rotterdam Oesophageal Tumor Study Group. Scand J Gastroenterol Suppl. 1998;225:75-84.

11. Grünberger B, Raderer M, Schmidinger M, Hejna M. Palliative chemotherapy for recurrent and metastatic esophageal cancer. Anticancer Res. 2007;27:2705-2714.

12. Amdal CD, Jacobsen AB, Guren MG, Bjordal K. Patient-reported outcomes evaluating palliative radiotherapy and chemotherapy in patients with oesophageal cancer: a systematic review. Acta Oncol. 2013;52:679-690.

13. Amdal CD, Jacobsen AB, Sandstad B, Warloe T, Bjordal K. Palliative brachytherapy with or without primary stent placement in patients with oesophageal cancer, a randomised phase III trial. Radiother Oncol. 2013;107:428-433.

14. Zhu HD, Guo JH, Mao AW, et al. Conventional stents versus stents loaded with (125)iodine seeds for the treatment of unresectable oesophageal cancer: a multicentre, randomised phase 3 trial. Lancet Oncol. 2014;15:612-619.

15. Javed A, Pal S, Dash NR, et al. Palliative stenting with or without radiotherapy for inoperable esophageal carcinoma: a randomized trial. J Gastrointest Cancer. 2012;43:63-69.

16. Rosenblatt E, Jones G, Sur RK, et al. Adding external beam to intra-luminal brachytherapy improves palliation in obstructive squamous cell oesophageal cancer: a prospective multi-centre randomized trial of the International Atomic Energy Agency. Radiother Oncol. 2010;97:488-494.

17. Ikeda E, Kojima T, Kaneko K, et al. Efficacy of concurrent chemoradiotherapy as a palliative treatment in stage IVB esophageal cancer patients with dysphagia. Jpn J Clin Oncol. 2011;41:964-72.

18. Murray LJ, Din OS, Kumar VS, Dixon LM, Wadsley JC. Palliative radiotherapy in patients with esophageal carcinoma: A retrospective review. Pract Radiat Oncol. 2012;2:257-264. 